\title{
Career management for scientists
}

\begin{abstract}
In the first of a monthly series of articles, written in the main by people who are professionally involved in recruitment, Larry Botheras sets out some ground rules for those considering a career move into industry.
\end{abstract}

THE management of their careers is probably something that sits close to the bottom of most people's lists of priorities while the job is going well. Unfortunately, it is most needed at times of 'distress', for example when funding is about to run out, or cuts are being imposed. Considering the way in which most careers start off, and the generally uninformed way in which one makes life-directing decisions at a young age, perhaps there is a much greater scope for proactivity.

To expand on the problems: typically, academic careers are initiated during school years, when, frequently, advice on careers is given by a teacher who has probably not spent any time out of an academic environment. This undoubtedly colours the advice given (the principle being to recommend what you know best) and it is further reinforced by any advice - both formal and informal - given at university level, which tends to be on the same basis. Unfortunately, for most people there is a time in their lives at which they find themselves bored with their role, or thinking that they have made the wrong choice and want a change. (For scientists, this is typically reflected in the desire to get away from the bench.) It is often the time when recruiters, such as myself, become involved in helping to pick up the pieces. This can be an extremely traumatic time for any individual, who may have little idea of how a portfolio of skills may be used in an alternative career.

The pharmaceutical and health care industry can be a highly beneficial provider of alternatives for the life science population, with a wide range of opportunities through which careers can evolve away from the bench. Typical routes for scientists moving into industry are clinical trials, regulatory affairs, project management, medical information or strategic product development. These areas are of critical importance to companies and call for a good scientific training, giving the new initiate 'street credibility'. Subsequent development will depend on the ability of the individual to interact well with a wide range of people with divergent interests.

These disciplines can be summarized as follows:

- Clinical trials - the management of studies carried out in man on new products. Typically this involves liaison with physicians and nurses at investigating centres (hospitals primarily), collection of the data and coordination of the trial.

- Regulatory affairs - liaison with governmental organizations to acquire a drug licence. This involves considerable contact with the clinical research team to ensure that the trial is likely to produce data of an acceptable standard.

- Project management - the overall management of a drug development programme, including timing, finance and resources. It will involve liaison with groups from the research, development, clinical, regulatory affairs, manufacturing and marketing areas.

- Medical information - answering written and verbal queries from both healthcare professionals (doctors, pharmacists) and the general public. It also includes maintenance of up-to-the-minute information on the competition as well as one's own products, including adverse reactions.

- Strategic product development - the assessment of new ideas, products and therapeutic areas to develop company R\&D strategy for the future. It involves working with marketing as well as senior management and R\&D.

There is, of course, a fairly major distrust of industry by those in academic institutions, in my view fuelled primarily by ignorance. The standard view of pharmaceutical research has been that options are limited, pressure is high and the drive for profitability is relentless. Certainly, research is more directed, but, in the end, very little research anywhere is done on an altruistic basis - everyone has to generate funding, and that always means project justification. It is a fact that a considerable amount of original medical science has been carried out by the pharmaceutical and healthcare industries, with a number of the most capable individuals at funded institutes winning Nobel prizes.

\section{Job hunting}

So you are persuaded - but what are the issues to be addressed in looking for a job in industry?

- Presentation - The first major area for improvement is usually the written career history. If applying for a research job in industry this is less applicable, but for other positions a serious rethink is important. The main criticism that I have is the tendency for academic CVs to be judged on thickness. The package needs, on the contrary, to be succinct, highlighting specific achievements rather than rambling through many details. Certainly include a list of publications, but if it is long, abridge it to include the most recent or most relevant. The recruiter, if interested, can then ask for a full list.

Second, try to put yourself in the position of the recruiter. He may have 200 CVs to look at, so try to stand out. That means focusing the application on the particular opportunity and ensuring that it looks as if time has been taken with the presentation.

- Where to apply - The advice here is relatively simple: start networking among colleagues and contacts, among whom there are bound to be people who have moved into the industry. Ask them to whom you could talk. The important thing about networking is not to put contacts under pressure. Do not ask them if they know of a vacancy - this puts them under pressure in two respects; through the possibility of letting you down, or through their reputation being put at risk within their company by recommending you.

Obviously, you must assiduously scan the appointments pages of journals. The obvious international journal is Nature, but there are also specific industry journals, the best known of which is Scrip published twice weekly. It is highly specialized, but it is a good place to see some relevant advertisements.

Finally, there are a number of specialist recruitment companies that concentrate on recruiting for the industry, so use your network contacts to find the most appropriate for your interests. Send a copy of your career history and wait for the response.

\section{Final thoughts}

Once you have started the networking process, do not give up on it the minute you find a job. Your network in its new, extended form is a valuable asset and needs to be nurtured. You will possibly need to use it on other occasions.

Over the past few years there has been a move away from the 'career for life' syndrome, so keep your mind open as far as possible. Remember that there is only a small gap between preconceptions and prejudices.

Larry Botheras is Head of Pharmaceuticals and Healthcare at Macmillan Davies, the executive search and selection consultancy based at Salisbury House, Bluecoats, Hertford, Herts, UK.

NATURE · VOL 367 • 24 FEBRUARY 1994 